Int. J. Odontostomat., 10(3):521-529, 2016.

\title{
Cáncer Oral y Dentistas: Conocimientos, Actitudes y Prácticas en Chile
}

\author{
Oral Cancer and Dentists: Knowledge, Attitudes and Practices in Chile
}

\author{
Stillfried A.; Rocha A." ; Colella G." \& Escobar E."
}

STILLFRIED, A.; ROCHA, A.; COLELLA, G. \& ESCOBAR, E. Cáncer oral y dentistas: conocimientos, actitudes y prácticas en Chile. Int. J. Odontostomat., 10(3):521-529, 2016.

RESUMEN: El objetivo de este estudio fue describir los conocimientos, actitudes y prácticas en relación al cáncer oral de Cirujano - Dentistas de la comuna Las Condes, Santiago de Chile. Se realizó un estudio descriptivo, transversal. Se aplicó una encuesta de autollenado a 264 Cirujano - Dentistas de la comuna Las Condes, Santiago de Chile en el período junio - julio 2014. Se informó previamente a los participantes el carácter voluntario, anónimo y confidencial de la encuesta. La muestra final fue de 205 (Tasa de pérdida: 22,3 \%).Se realizó estadística descriptiva con tabulación y distribución de frecuencias. La mayoría de los participantes identificó los principales factores de riesgo decáncer oral: tabaquismo (99,5\%) y consumo de alcohol (83,4 \%). Menos de dos tercios identificó las dos lesiones cancerizables más frecuentes (leucoplasia y eritroplasia) y al carcinoma espinocelular como la forma más común de cáncer oral. El 25,4 \% reportó presentar conocimientos actualizados sobre el cáncer oral y el 35,6 \% reportó practicar habitualmente un examen clínico para detectar cáncer oral. Se observaron carencias en los conocimientos, actitudes y prácticas en relación al cáncer oral de Cirujano Dentistas de la comuna Las Condes, Santiago de Chile. Estas carencias son susceptibles a ser mejoradas mediante intervenciones educacionales dirigidas a Cirujano - Dentistas que permitan mejorar sus conocimientos, actitudes preventivas y competencias procedimentales en la prevención y diagnóstico precoz del cáncer oral.

PALABRAS CLAVE: cáncer oral, cirujano dentistas, conocimiento, actitudes, prácticas, comportamiento, chile, prevención, factores de riesgo.

\section{INTRODUCCIÓN}

El cáncer oral es un problema de salud pública a nivel mundial, con tasas de incidencia y mortalidad en aumento (Kujan et al., 2006). Según informes mundiales, es el sexto cáncer más común, presentándose 575.000 casos nuevos y 200.000 muertes anualmente (Noonan, 2014). Más del $90 \%$ de las neoplasias malignas de la cavidad oral corresponden a carcinoma espinocelular oral (CECO) (Rhodus, 2014). A pesar de los avances en los métodos diagnósticos y modalidades terapéuticas, el CECO presenta una de las tasas de supervivencia más bajas a los 5 años, la cual ha permanecido inalterada durante los últimos 50 años, siendo aún de un 50-55 \% (Kujan et al.). Alrededor de un $70 \%$ de los pacientes con CECO son diagnosticados en estadios avanzados de la enferme- dad (estadios III y IV), cuando el paciente ya presenta dolor e impotencia funcional (Patel et al., 2011). El CECO diagnosticado en etapas avanzadas requiere de un tratamiento radical y se asocia con un mal pronóstico (Noonan), mientras que el CECO diagnosticado en estadios tempranos requiere de un tratamiento menos invasivo y se asocia a mejores tasas de supervivencia (Abdullah, 2011 \& Morse et al., 2011). Es así, como la detección precoz del CECO, se considera la medida más efectiva en la reducción de su morbimortalidad (Noonan). Se postula que los escasos cambios que se han observado en la tasa de supervivencia, se deben al frecuente retraso diagnóstico del CECO (Rocha-Buelvas et al., 2012). Se describen en la literatura dos causas principales: la falta de conoci-

\footnotetext{
* Carrera de Odontología, Facultad de Medicina - Clínica Alemana Universidad del Desarrollo

* Universidad Nacional de Colombia, Colombia

*** Universidad de Nápoles, Italia

**** Departamento de Patología y Medicina Oral, Facultad de Odontología, Universidad de Chile
} 
miento de la población sobre el CECO y sus factores de riesgo asociados y la falta de conocimiento y la actitud inadecuada de los odontólogos en relación al CECO (Joseph et al., 2012 \& M et al., 2013). Los odontólogos cumplen un rol crucial en la prevención primaria y secundaria del CECO, teniendo el deber de informar a sus pacientes sobre los factores de riesgo asociados y de realizar un examen clínico sistemático de la cavidad oral en forma rutinaria, permitiendo su detección precoz (Hertrampf et al., 2012). Por lo que se requiere que presenten conocimientos, actitudes y prácticas adecuadas para identificar pacientes con estilos de vida poco saludables que incrementan considerablemente el riesgo de desarrollo de cáncer y para la ejecución de un examen clínico sistemático de la cavidad oral (Abdullah). Diversos estudios internacionales han evaluado los conocimientos, actitudes y prácticas de los odontólogos en relación al CECO. Los resultados de estos estudios difieren y la información específica de cada país es necesaria para la planificación en salud pública, ya que los programas de prevención del CECO deben diseñarse acorde a las condiciones y necesidades concretas del lugar geográfico en que se implementan (Colella et al., 2008). Por lo que, previo al desarrollo e implementación de programas educacionales para odontólogos para la prevención del CECO es imprescindible la evaluación de sus conocimientos, actitudes y prácticas en relación al CECO (Morales et al., 2009). En la actualidad, no existen estudios de este tipo publicados en Chile, lo que nos motivó a realizar este estudio con el objetivo de describir los conocimientos, actitudes y prácticas en relación al cáncer oral de Cirujano - Dentistas de la comuna Las Condes, Santiago de Chile.

\section{MATERIAL Y MÉTODO}

Se realizó un estudio descriptivo, transversal que consistió en la aplicación de la encuesta de auto llenado: "Conocimientos, actitudes y prácticas de los Odontólogos en relación al cáncer oral" validada y aplicada por Colella et al. y traducida al Castellano y aplicada por Rocha-Buelvas et al. La encuesta consta de un total de 31 preguntas cerradas con respuestas de tipo dicotómicas y policotómicas. La encuesta incluye preguntas relativas a las características demográficas y profesionales de los participantes, al conocimiento en relación a factores de riesgo y procedimientos diagnósticos del cáncer oral y en relación a las actitudes y prácticas respecto al cáncer oral. La encuesta se sometió a una prueba piloto previo a su aplicación, con el fin de evaluar la comprensión de los contenidos, el tiempo de ejecución y las carencias temáticas que los participantes consideraran relevantes para asegurar la interpretación adecuada de las preguntas. En base a los comentarios obtenidos de los participantes de la prueba piloto, se revisó la encuesta en cuanto a redacción, comprensión de contenidos y formato, y se introdujeron las correspondientes modificaciones. La encuesta se entregó a 264 Cirujano Dentistas de la comuna Las Condes, Santiago de Chile en el período junio - julio 2014. Se incluyeron a Cirujano - Dentistas de nacionalidad Chilena y de práctica activa pública o privada en la comuna Las Condes, Santiago de Chile. Se excluyeron a Cirujano Dentistas que trabajen exclusivamente en el área administrativa o no se dediquen profesionalmente al ámbito de la Odontología.

Se realizó la entrega personal de la encuesta a los participantes y se dio un período de 24 horas antes de su retiro. Se informó previamente a los participantes el carácter voluntario, anónimo y confidencial de la encuesta, durante y después de la investigación. La encuesta se entregó a los participantes dentro de un sobre, que además de la encuesta contenía una hoja de instrucciones para los participantes, un consentimiento informado y otro sobre. Los participantes entregaron la encuesta y el consentimiento informado en los dos sobres independientes sellados a colaboradores del estudio encargados del retiro. Ambos documentos se mantuvieron bajo estricta confidencialidad de los datos y anonimizados. La información obtenida a través de la encuesta y el consentimiento informado se disoció irreversiblemente.

De un total de 264 encuestas entregadas a $\mathrm{Ci}$ rujano - Dentistas de la comuna Las Condes, 47 encuestas no fueron devueltas y de las 217 encuestas restantes, 12 se excluyeron del estudio por presentar el autollenado de respuestas de manera incompleta. La tasa de pérdida fue del $22,3 \%$, por lo que la muestra final fue de 205. Los resultados se ingresaron en una plantilla Microsoft Excel® 2011. Se realizó estadística descriptiva, para las variables cuantitativas discretas con medias y desviación estándar y para las variables categóricas nominales con tabulación y distribución de frecuencias: frecuencia absoluta y porcentaje. Para estos análisis se utilizó el programa Microsoft Excel $囚$ versión 2011. El estudio fue aprobado por el Comité de Ética de la investigación de la Facultad de Medicina Clínica Alemana - Universidad del Desarrollo, Chile. 


\section{RESULTADOS}

Características demográficas y profesionales. La distribución de las características demográficas y profesionales de los participantes se detalla en la Tabla I. Del total de participantes, se registró una cantidad levemente mayor de hombres $(51,2 \%)$ que de mujeres. El $41 \%$ eran odontólogos generales y $59 \%$ especialistas, los cuales presentaron un promedio de 10,5 años desde su titulación. Las especialidades más comunes fueron periodoncia (25\%) y rehabilitación oral $(25 \%)$. En cuanto al tipo de práctica profesional, en el tipo dependiente, el $32,2 \%$ se desempeñaba profesionalmente en el sistema privado y el $17,6 \%$ en el sistema público. En relación al tiempo transcurrido desde el último curso o actualización en cáncer oral, el $43,4 \%$ de los participantes reportó nunca haber hecho un curso de capacitación en cáncer oral.

\section{Conocimientos en relación a factores de riesgo de} cáncer oral. El 99,5\% de los participantes reconoció el tabaquismo y la presencia de lesiones precancerosas como factores de riesgo de cáncer oral. Alrededor del $80 \%$ identificó como factores de riesgo el consumo de alcohol y la exposición al sol en cáncer de labio. La mitad de los participantes reconoció la edad avanzada como factor de riesgo y sólo el 15,6 \% relacionó el bajo consumo de frutas y verduras con un riesgo aumentado de cáncer oral (Tabla II). En general, aquellos factores que no son considerados factores de riesgo de cáncer oral fueron identificados por menos participantes (Fig. 1).
Conocimientos en relación a procedimientos diagnósticos de cáncer oral. El $58 \%$ de los participantes identificó el carcinoma espinocelular como la forma más común de cáncer oral. El 63,9 \% identificó a la leucoplasia y la eritroplasia como las dos lesiones cancerizables más frecuentes, sin embargo, sólo el $41,2 \%$ de éstos conocía que la eritroplasia presenta un mayor potencial de malignización que la leucoplasia. El 57,1\% reconoció la ulceración como la manifestación inicial más frecuente de cáncer oral y sólo el 43,4 $\%$ identificó los sitios más frecuentes en los que se desarrolla. El $60,5 \%$ de los participantes conocía que el cáncer oral es diagnosticado con mayor frecuencia en estadio avanzado y el $49,3 \%$ que es diagnosticado con mayor frecuencia entre los 40 y 59 años (Tabla III).

Actitudes en relación al cáncer oral. Las actitudes en relación al cáncer oral de los participantes se detaIlan en la Tabla IV. Sólo el $25,4 \%$ estuvo de acuerdo en presentar conocimientos actualizados sobre el cáncer oral. El $78 \%$ reportó estar adecuadamente preparado para explicar los riesgos del tabaquismo a nivel oral y el $57,1 \%$ para explicar los riesgos del consumo de alcohol a nivel oral. Pocos participantes reportaron que sus pacientes conocían los factores de riesgo de cáncer oral $(30,7 \%)$ y que conocían sus signos y síntomas $(9,8 \%)$. El 43,9 \% reportó estar adecuadamente preparado para efectuar un examen diagnóstico clínico de cáncer oral y el 60,5\% para realizar palpación de linfonodos cervicales.

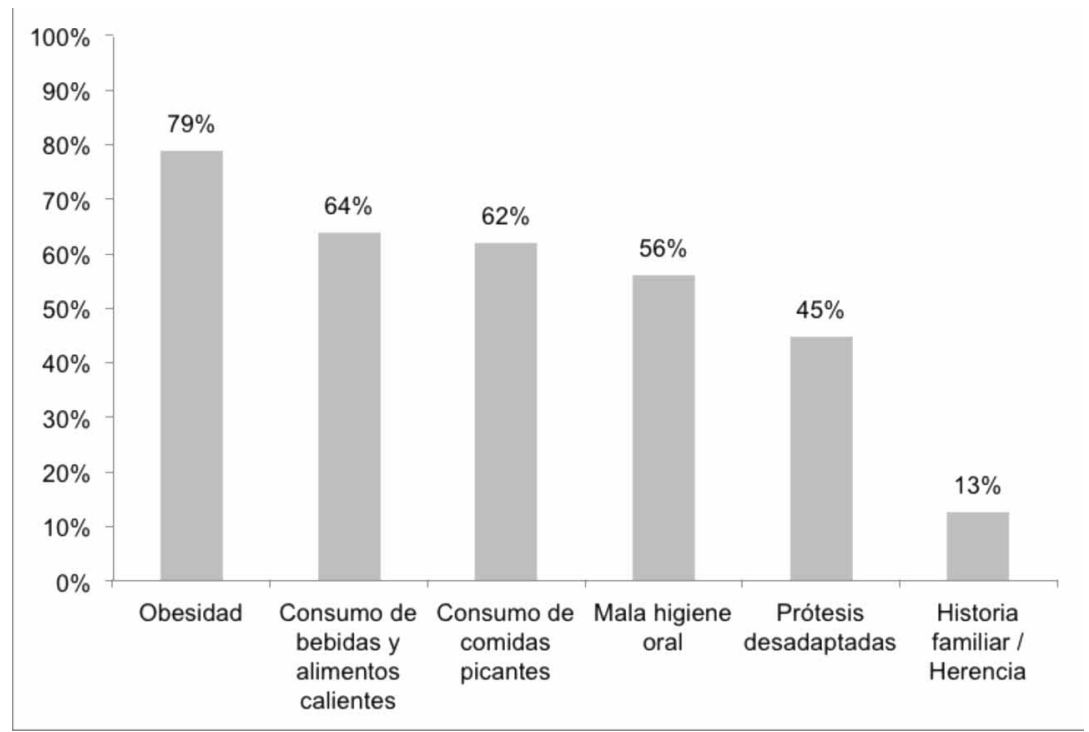

Fig. 1. Identificación de factores que no son considerados de riesgo de cáncer oral. 
Tabla I. Características demográficas y profesionales

\begin{tabular}{|c|c|c|c|}
\hline & $\mathbf{N}$ & $\%$ & - \pm \\
\hline Femenino & 100 & 48,8 & \\
\hline \multirow[t]{2}{*}{ Masculino } & 105 & 51,2 & \\
\hline & & & $10,5 \pm 9,6$ \\
\hline $0-5$ & 69 & 33,7 & \\
\hline $6-10$ & 44 & 21,5 & \\
\hline $11-15$ & 19 & 9,3 & \\
\hline $16-20$ & 23 & 11,2 & \\
\hline$>20$ & 50 & 24,2 & \\
\hline Sin especialidad: Odontología General & 84 & 41,0 & \\
\hline Periodoncia & 25 & 12,2 & \\
\hline Rehabilitación Oral & 25 & 12,2 & \\
\hline Endodoncia & 23 & 11,2 & \\
\hline Implantología & 16 & 7,8 & \\
\hline Odontopediatría & 10 & 4,9 & \\
\hline Ortodoncia y Ortopedia DentoMáxilo Facial & 10 & 4,9 & \\
\hline Radiología DentoMáxilo Facial & 6 & 2,9 & \\
\hline Cirugía y Traumatología Bucal y Máxilo Facial & 4 & 2,0 & \\
\hline Patología Buco Máxilo Facial & 2 & 1,0 & \\
\hline Dependiente / sistema privado & 66 & 32,2 & \\
\hline Dependiente / sistema público & 36 & 17,6 & \\
\hline Independiente / autónomo & 61 & 29,8 & \\
\hline Independiente / en sociedad & 42 & 20,5 & \\
\hline$<12$ meses & 26 & 12,7 & \\
\hline $1-4$ años & 50 & 24,4 & \\
\hline$>5$ años & 40 & 19,5 & \\
\hline Nunca he hecho un curso de capacitación en cáncer oral & 89 & 43,4 & \\
\hline
\end{tabular}

Tabla II. Conocimientos en relación a factores de riesgo de cáncer oral

\begin{tabular}{lcccccc}
\hline & \multicolumn{2}{c}{$\mathrm{Si}$} & \multicolumn{2}{c}{ No } & \multicolumn{2}{c}{ No sé } \\
& $\mathrm{N}$ & $\%$ & $\mathrm{~N}$ & $\%$ & $\mathrm{~N}$ & $\%$ \\
\hline Factores de riesgo & & & & & & \\
Tabaquismo & 204 & 99,5 & 1 & 0,5 & 0 & 0,0 \\
Presencia de lesiones precancerosas & 204 & 99,5 & 1 & 0,5 & 0 & 0,0 \\
Consumo de alcohol & 171 & 83,4 & 15 & 7,3 & 19 & 9,3 \\
Exposición al sol en cáncer de labio & 166 & 81,0 & 30 & 14,6 & 9 & 4,4 \\
Edad avanzada & 104 & 50,7 & 80 & 39,0 & 21 & 10,2 \\
Bajo consumo de frutas y verduras & 32 & 15,6 & 128 & 62,4 & 45 & 22,0 \\
\hline
\end{tabular}

Prácticas en relación al cáncer oral. El 35,6 \% de los participantes reportó practicar habitualmente un examen diagnóstico clínico para detectar lesiones precancerizables y cáncer oral. El 64,4\% reportó no practicar dicho examen en forma habitual e indicó el motivo principal por el que no lo realiza (Fig. 2). Sólo el $41,5 \%$ de los participantes reportó realizar palpación de linfonodos cervicales a sus pacientes. De aquellos participantes que reportaron realizar habitualmente un examen diagnóstico clínico para detectar lesiones precancerizables y cáncer oral, el 57,5\% reportó realizar palpación ganglionar a sus pacientes (Tabla V).

Los aspectos considerados por los participantes dentro de la anamnesis remota personal de sus pacientes se detallan en la Tabla VI. El 94,6\% reportó preguntar a sus pacientes por el consumo actual de tabaco y el $82,9 \%$ por el tipo y cantidad de tabaco 
consumido. El 87,8 \% reportó preguntar a sus pacientes por el consumo actual de alcohol y el $66,8 \%$ por el tipo, cantidad y frecuencia de alcohol consumido. E
$77,1 \%$ y el $58,5 \%$ de los participantes reportó consultar por el consumo previo de tabaco y el consumo previo de alcohol.

Tabla III. Conocimientos en relación a procedimientos diagnósticos de cáncer oral.

\begin{tabular}{|c|c|c|c|c|}
\hline \multirow[b]{2}{*}{ Procedimientos diagnósticos } & \multicolumn{2}{|c|}{ Sabe } & \multicolumn{2}{|c|}{ No sabe } \\
\hline & $\mathrm{N}$ & $\%$ & $\mathrm{~N}$ & $\%$ \\
\hline $\begin{array}{l}\text { Lesiones orales precancerosas pequeñas, asintomáticas y eritematosas se asocian a una alta } \\
\text { probabilidad de progresión a cáncer oral }\end{array}$ & 102 & 49,8 & 103 & 50,2 \\
\hline La eritroplasia y la leucoplasia son las dos lesiones cancerizables más frecuentes & 131 & 63,9 & 74 & 36,1 \\
\hline El carcinoma espinocelular es la forma más común de cáncer oral & 119 & 58,0 & 86 & 42,0 \\
\hline El cáncer es diagnosticado con mayor frecuencia entre los 40 y 59 años & 101 & 49,3 & 104 & 50,7 \\
\hline La lengua y el piso de boca son los sitios más frecuentes en los que se desarrolla cáncer oral & 89 & 43,4 & 116 & 56,6 \\
\hline Los bordes laterales de la lengua son las superficies con mayor probabilidad de desarrollar cáncer oral & 133 & 64,9 & 72 & 35,1 \\
\hline La ulceración es la manifestación inicial más frecuente de cáncer oral & 117 & 57,1 & 88 & 42,9 \\
\hline En presencia de cáncer oral los linfonodos usualmente son pétreos, indoloros y fijos & 152 & 74,1 & 53 & 25,9 \\
\hline Las lesiones de cáncer oral son diagnosticadas más frecu entemente en estadio avanzado & 124 & 60,5 & 81 & 39.5 \\
\hline
\end{tabular}

Tabla IV. Actitudes en relación al cáncer oral

\begin{tabular}{|c|c|c|c|c|c|c|}
\hline & \multicolumn{2}{|c|}{ De acuerdo } & \multicolumn{2}{|c|}{ En desacuerdo } & \multicolumn{2}{|c|}{ No sé } \\
\hline & $\mathrm{N}$ & $\%$ & $\mathrm{~N}$ & $\%$ & $\mathrm{~N}$ & $\%$ \\
\hline Mis conocimientos sobre el cáncer oral son actualizados & 52 & 25,4 & 125 & 61,0 & 28 & 13,7 \\
\hline En presencia de lesiones orales sospechosas aconsejo al paciente realizar biopsia & 182 & 88,8 & 20 & 9,8 & 3 & 1,5 \\
\hline Mis pacientes están informados sobre los factores de riesgo de cáncer oral & 63 & 30,7 & 107 & 52,2 & 35 & 17,1 \\
\hline Mis pacientes conocen los signos y síntomas de cáncer oral & 20 & 9,8 & 146 & 71,2 & 39 & 19,0 \\
\hline $\begin{array}{l}\text { Estoy adecuadamente preparado para explicar los riesgos del tabaquismo a nivel } \\
\text { oral }\end{array}$ & 160 & 78,0 & 37 & 18,0 & 8 & 3,9 \\
\hline $\begin{array}{l}\text { Estoy adecuadamente preparado para explicar los riesgos del consumo de alcohol a } \\
\text { nivel oral }\end{array}$ & 117 & 57,1 & 76 & 37,1 & 12 & 5,9 \\
\hline $\begin{array}{l}\text { Estoy adecuadamente preparado para efectuar un examen diagnóstico clínico de } \\
\text { cáncer oral }\end{array}$ & 90 & 43,9 & 89 & 43,4 & 26 & 12,7 \\
\hline Estoy adecuadamente preparado para realizar palpación de linfonodos cervicales & 124 & 60,5 & 64 & 31,2 & 17 & 8,3 \\
\hline
\end{tabular}

Tabla V. Porcentaje de participantes que realiza examen diagnóstico clínico de cáncer oral y palpación de linfonodos.

\begin{tabular}{lccc}
\hline & & \multicolumn{2}{c}{ Realiza palpación de linfonodos } \\
\hline & & Si & No \\
Realiza examen diagnóstico clínico para detectar cáncer oral & Si & $57,5 \%$ & $42,5 \%$ \\
\hline
\end{tabular}

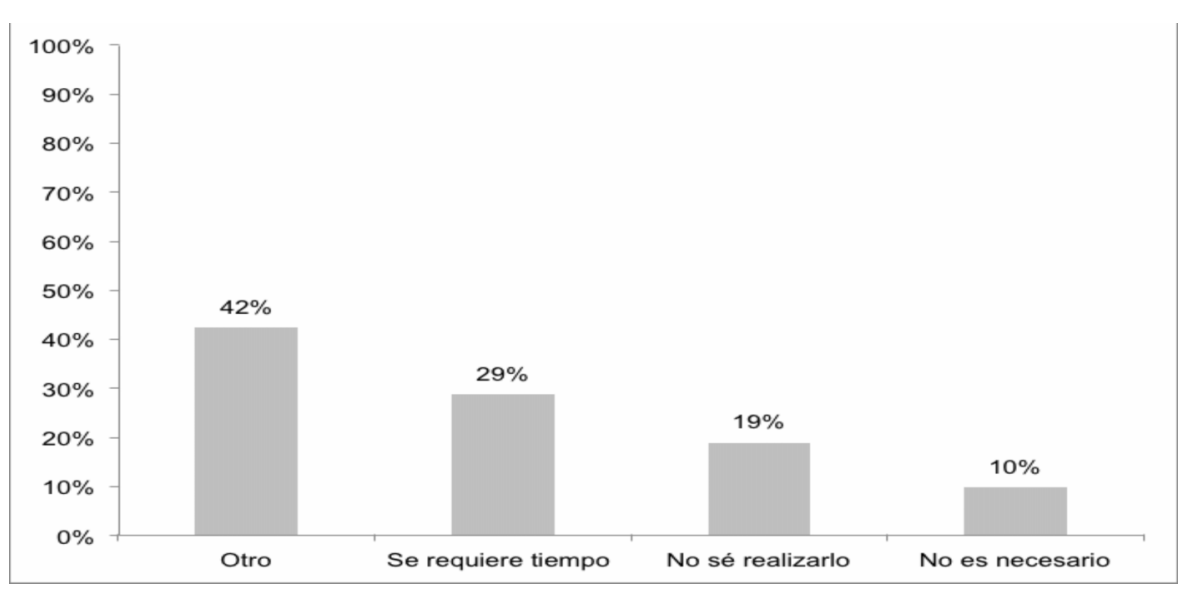

Fig. 2. Examen diagnóstico de cáncer oral de parte de los Cirujanos Dentistas. 


\section{DISCUSIÓN}

El presente estudio entrega una descripción del estado actual de conocimientos, actitudes y prácticas en relación al cáncer oral de Cirujano - Dentistas de la comuna Las Condes, Santiago de Chile. Conocer los conocimientos, actitudes y prácticas de Cirujano Dentistas es fundamental para evaluar su efectividad en la prevención y detección precoz del cáncer oral, y por ende, en la reducción de su morbi-mortalidad (Kujan et al.) En la actualidad, no existen estudios de este tipo publicados en Chile.

El diagnóstico precoz del cáncer oral es un objetivo prioritario de salud pública, en el cual los odontólogos deben desempeñar un papel protagonista. La detección del cáncer oral en un estadio precoz, garantiza no sólo un aumento de las tasas de supervivencia, sino también una mejor calidad de vida, ya que permite la aplicación de medidas terapéuticas más conservadoras y una respuesta más favorable al tratamiento (Kujan et al.) Es así, como esta medida se considera la más efectiva en la reducción de su morbi - mortalidad (Noonan). Diversos estudios en Estados Unidos, Canadá, Europa y Arabia Saudita han descrito los conocimientos, actitudes y prácticas de odontólogos en relación al cáncer oral. Estos mismos estudios sugieren que los odontólogos no detectan lesiones orales de manera oportuna debido a una falta de conocimiento o actitudes y prácticas inadecuadas (Abdullah; Ergun et al., 2009; Hertrampf et al. 2010a).

Las comparaciones de los resultados obtenidos en este estudio con los de otros estudios realizados en distintos países son interesantes, pero requieren de precaución en las interpretaciones de éstos. Se debe considerar que son múltiples los factores que pueden influir en los conocimientos, actitudes y prácticas de odontólogos y se deben tener en cuenta diferencias importantes, tales como las características demográficas y profesionales de la población estudiada, los diferentes métodos de recolección de datos y de medición de resultados. La comparación de los resultados de este estudio con los de otros estudios internacionales demuestra que los valores obtenidos en general fueron inferiores. Los Cirujano - Dentistas deben presentar adecuados conocimientos en relación a los factores de riesgo de cáncer oral para poder identificar a aquellos individuos en riesgo y poder asesorarlos.

En este estudio, casi la totalidad de los CirujanoDentistas reconoció el tabaquismo y la presencia de le- siones precancerosas como factores de riesgo de cáncer oral y alrededor del $80 \%$ identificó también el consumo de alcohol. La edad avanzada fue identificada por un menor número de participantes y muy pocos conocían el efecto protector de las frutas y verduras, ya que sólo el 15,6 \% relacionó su bajo consumo con un riesgo aumentado de cáncer oral. Estos hallazgos son muy similares a los de otros estudios internacionales (Yellowitz et al., 2000; Canto et al., 2001 \& Hertrampf et al., 2010b). En general, aquellos factores que no son considerados factores de riesgo de cáncer oral fueron identificados por menos participantes. Más de la mitad reportó incorrectamente el uso de prótesis desadaptadas como factor de riesgo y casi el $90 \%$ la historia familiar. Otros estudios (Alonge et al., 2003; Patton et al., 2006 Hertrampf et al., 2010b) también han observado esta discrepancia en el conocimiento en relación a factores de riesgo versus factores de no riesgo.

En los ítems de conocimientos en relación a procedimientos diagnósticos de cáncer oral se encontraron carencias importantes. Sólo el $58 \%$ de los participantes identificó el carcinoma espinocelular como la forma más común de cáncer oral. Este hallazgo fue inferior al valor obtenido por la mayoría de los estudios (Yellowitz et al., 1998; Yellowitz et al., 2000; Canto et al.; Clovie et al., 2002; Alonge et al.; Patton et al. \& Hertrampf et al., 2010b) cuyo rango fue del $82 \%$ al 87 $\%$, sin embargo, fue levemente superior al valor obtenido por Colella y en Italia (51\%) y por Rocha - Buelvas y cols. en Colombia (52 \%) (Colella et al. \& RochaBuelvas et al.).

Sólo dos tercios de los Cirujano-Dentistas encuestados identificaron a la leucoplasia y la eritroplasia como las dos lesiones cancerizables más frecuentes, siendo este hallazgo inferior al reportado por Yellowitz et al. y Clovis et al. (2002). De aquellos que identificaron correctamente las dos lesiones cancerizables más frecuentes, sólo el 41,2\% conocía el mayor potencial de malignización de la eritroplasia respecto a la leucoplasia. Este hallazgo sugiere que los CirujanoDentistas enfatizan el reconocimiento y diagnóstico de lesiones de apariencia leucoplásica en desmedro de lesiones de apariencia eritroplásica, lo cual es relevante, ya que el potencial de transformación maligna de las eritroplasias es superior al de las leucoplasias (Reichart et al., 2005).

En este estudio sólo el 43,4 \% identificó la lengua y el piso de la cavidad oral como los sitios en los que se desarrolla más frecuentemente el cáncer oral, esto fue similar a lo reportado por Alonge et al. (51\%) 
y Yellowitz (54 \%), sin embargo, mayor a lo reportado por Colella (32\%) y Rocha-Buelvas (18\%) (Patton et al.; Patel et al. \& Hertrampf et al., 2013). Esta cifra es importante destacar, ya que refleja que más de la mitad de los odontólogos no conoce los sitios más frecuentes para cáncer oral.

El $74,1 \%$ de los Cirujano - Dentistas encuestados conocía las características de una linfoadenopatíamaligna: linfonodos pétreos, indoloros y fijos. Canto et al. y Patton et al. reportaron un porcentaje similar (76 \% y $70 \%$ respectivamente) (Canto el al. \& Patton et al.). A pesar de que este valor es superior al obtenido en los otros ítems de conocimiento, se debe destacar, ya que uno de cada cuatro Cirujano - Dentistas no presentaría las competencias adecuadas para diferenciar una linfoadenopatía cervical maligna de una benigna. Sólo el $25,4 \%$ de los odontólogos declaró presentar conocimientos actualizados sobre el cáncer oral, siendo esta cifra inferior a la reportada por Clovis et al. en su estudio realizado en Canadá (Clovis et al.). Este hallazgo sugiere que la gran mayoría de los odontólogos reconoce sus carencias de conocimiento en relación al cáncer oral. Pocos odontólogos reportaron que sus pacientes conocían los factores de riesgo y los signos y síntomas del cáncer oral, 30,7 \% y $9,8 \%$ respectivamente. Esto refleja que los Cirujano Dentistas no informan a sus pacientes sobre los factores de riesgo de cáncer oral, sobre sus signos y síntomas, ni tampoco sobre la importancia del autoexamen de la cavidad oral y de consultar en caso de detectar una lesión oral sospechosa.

Los resultados de este estudio indican que realizar habitualmente un examen clínico diagnóstico de cáncer oral no es una práctica común de Cirujano - Dentistas, ya que sólo el $35,6 \%$ reportó realizarlo. Este valor es aún menor al obtenido por Colella et al. en su estudio realizado en Italia (Colella et al.). Este hallazgo es importante, ya que indica que en muchos pacientes podrían pasar desapercibidas lesiones orales tanto premalignas como malignas por falta de un examen clínico sistemático de la cavidad oral, es decir, una actitud inadecuada de sus odontólogos tratantes. Además es posible que algunos de los odontólogos que reportaron efectuar habitualmente un examen clínico diagnóstico de cáncer oral, no presenten las competencias suficientes para realizarlo en forma efectiva.

Muchos odontólogos presentaron conocimientos inadecuados en relación a los sitios más frecuentes y las lesiones precancerizables más comunes (dónde y qué buscar), sólo el 43,9\% reportó estar adecuadamente preparado para realizarlo y de los que reportaron practicarlo, un $42,5 \%$ no realiza palpación de los linfonodos a sus pacientes, omitiendo una etapa crítica y fundamental del examen clínico para detectar cáncer oral. EI $60,5 \%$ de los Cirujano - Dentistas declara estar adecuadamente preparado para realizar palpación de linfonodos cervicales, sin embargo, sólo el 41,5\% la realiza. Esta discrepancia entre la actitud y la práctica puede significar que a pesar de que conozcan las maniobras técnicas para realizar la palpación, no la realizan como parte integral del examen clínico.

Los odontólogos deben consignar en la anamnesis remota personal toda la información concerniente a factores de riesgo de cáncer oral y, de esta manera, identificar a los pacientes de alto riesgo. En este estudio el porcentaje de odontólogos que considera dentro de la anamnesis remota personal el consumo actual y previo de tabaco $(94,6 \%$ y $77,1 \%)$ y el consumo actual y previo de alcohol ( $87,8 \%$ y $58,5 \%)$ es comparable con los resultados obtenidos por Colella et al. en Italia. En ambos estudios son más los odontólogos que consultan por el consumo actual de tabaco y alcohol que los que consultan por el consumo previo de estas sustancias (Colella et al.) Al no indagar sobre el consumo previo de tabaco o de alcohol, se podría subestimar el riesgo de pacientes ex fumadores o ex - bebedores, especialmente de aquellos que han abandonado recientemente el hábito.

Los resultados de este estudio deben interpretarse en el contexto de sus limitaciones metodológicas. Al utilizar como instrumento de medición una encuesta de autollenado, es decir, el autoreporte de datos, existe la posibilidad de que las prácticas reportadas por los participantes no coincidan con su práctica clínica real. La tendencia de los participantes a dar respuestas socialmente aceptadas en las variables de práctica puede eventualmente haber generado un sesgo de información. Sin embargo, el carácter anónimo de la encuesta puede haber contribuido a minimizar este sesgo. Es probable que los participantes de este estudio presenten un mayor conocimiento e interés en el tópico cáncer oral que aquellos que rechazaron participar en él, por lo que los resultados podrían subestimar las carencias reales de conocimiento en relación al cáncer oral. Este tipo de sesgo ha sido sugerido también por otros autores (Clovis et al.).

Este estudio entrega evidencia que sugiere la necesidad de desarrollo e implementación de programas educacionales para Cirujano - Dentistas chile- 
nos, que permitan capacitarlos en el tópico cáncer oral y que permitan aumentar su eficacia en la prevención y diagnóstico precoz de esta enfermedad. Estas intervenciones educacionales podrían ser efectivas para mejorar o corregir las carencias que reveló este estudio en cuanto a conocimientos, actitudes y prácticas en relación al cáncer oral. Un estudio desarrollado por Hertrampf et al. en Alemania, demostró que las intervenciones educacionales son eficaces en mejorar las competencias de Cirujano - Dentistas para la detección precoz del cáncer oral. La recomendación es que asistan regularmente a este tipo de cursos de actualización (Hertrampf et al., 2013). Otra estrategia importante para asegurar en el país futuros Cirujano - Dentistas con adecuadas competencias en relación al cáncer oral, es evaluar y mejorar los contenidos curriculares de pregrado de las distintas escuelas de Odontología relacionados a las competencias cognitivas y procedimentales en el diagnóstico de precáncer y cáncer oral.

\section{CONCLUSIONES}

En este estudio se observaron carencias en los conocimientos, actitudes y prácticas en relación al cáncer oral de Cirujano - Dentistas de la comuna Las Condes, Santiago de Chile.Estas carencias son susceptibles a ser mejoradas mediante intervenciones educacionales dirigidas a Cirujano - Dentistas que permitan mejorar sus conocimientos, actitudes preventivas y competencias procedimentales en la prevención y diagnóstico precoz del cáncer oral. La formación de Cirujano - Dentistas adecuadamente capacitados en el diagnóstico precoz del cáncer oral, es crucial como medida para la reducción de la morbi mortalidad por esta enfermedad.

AGRADECIMIENTOS. A todas aquellas personas que de una u otra forma, colaboraron o participaron en esta investigación.

STILLFRIED, A.; ROCHA, A.; COLELLA, G. \& ESCOBAR, E. Oral cancer and dentists: knowledge, attitudes and practices in Chile. Int. J. Odontostomat., 10(3):521$529,2016$.

ABSTRACT: The aim of this survey was to describe the knowledge, attitudes and practices regarding oral cancer in a sample of dentists from Las Condes, Santiago de Chile. A descriptive, cross-sectional study was conducted. A self-filling survey was applied to 264 dentists of Las Condes, Santiago de Chile during june - july 2014. Participants were previously informed that the survey was voluntary, anonymous and confidential. The final sample included 205 dentists (Loss Rate: $22.3 \%$ ). Descriptive statistic was applied using tabulation and frequency distribution. Most participants identified the main risk factors for oral cancer: tobacco (99.5\%) and alcohol usage (83.4 $\%)$. Less than two thirds of the participants identified the two most common precancerous lesions (leukoplakia and erythroplakia) and squamous cell carcinoma as the most common type of oral cancer. $25.4 \%$ of the participants reported to have updated knowledge regarding oral cancer and $35.6 \%$ reported to routinely perform clinical examination to detect oral cancer. Deficiencies in knowledge, attitudes and practices regarding oral cancer of dentists from Las Condes, Santiago de Chile were observed. These deficiencies are likely to be enhanced through educational interventions which would improve their knowledge, attitudes and strengthen their role in the prevention and early diagnosis of oral cancer.

KEY WORDS: oral cancer, dentists, knowledge, attitudes, practices, behavior, Chile, prevention, risk factors

\section{REFERENCIAS BIBLIOGRÁFICAS}

Abdullah-Jaber, M. Dental practitioner'sknowledge, opinions and methods of management of oral premalignancy and malignancy. Saudi Dental J., 23(1):29-36, 2011.

Alonge, O.K. \& Narendran, S. Opinions about oral cancer prevention and early detection among dentists practicing along the Texas-Mexicoborder. Oral Diseases, 9(1):415, 2003.

Canto, M. T.; Drury, T. F. \& Horowitz, A. M. Maryland Dentists' Knowledge of Oral Cancer Risk Factors and Diagnostic Procedures. Health Promotion Practice, 2(3):255-262, 2001.

Clovis, J. B.; Horowitz, A. M. \& Poel, D. H. Oral and pharyngealcancer: practices and opinions of dentists in British Columbia and Nova Scotia. J. Canadian Dental Association, 68(7):421-425, 2002.

Colella, G.; Gaeta, G. M.; Moscariello, A. \& Angelillo, I. F. Oral cancer and dentists: Knowledge, attitudes, and practices in Italy. Oral Oncology, 44(4):393-9, 2008.

Ergun, S.; Özel, S.; Koray, M.; Kürklü, E.; Ak, G. \& Tanyeri, H. Dentists' knowledge and opinions about oral mucosal lesions. Int. J. Oral and Maxillofacial Surg., 38(12):12838, 2009. 
Hertrampf, K.; Wiltfang, J.; Koller, M.; Klosa, K. \& Wenz, H. J. Dentists' perspectives on oral cancer: a survey in Northern Germany and a comparison with international data. European Journal of Cancer Prevention: TheOfficial J. European Cancer Prevention Organization (ECP), 19(2):144-52, 2010.

Hertrampf, K.; Wenz, H.-J.; Koller, M. \& Wiltfang, J. Comparing dentists' and the public's awareness about oral cancer in a community-based study in Northern Germany. J. Cranio-Maxillo-Facial Surg., 40(1):28-32, 2012.

Hertrampf, K.; Wenz, H.-J.; Koller, M.; Grund, S. \& Wiltfang, J. Early detection of oral cancer: dentists' opinions and practices before and after educational interventions in Northern-Germany. Journal of Cranio-Maxillo-Facial Surgery: Official Publication of the European Association for Cranio-Maxillo-Facial Surgery, 41(8):201-7, 2013.

Joseph, B. K.; Sundaram, D. B. \& Sharma, P. Oral cancer awareness among dentists in Kuwait. Medical Principles and Practice: International Journal of the Kuwait University, Health Science Centre, 21(2):164-70, 2012.

Kujan, O.; Duxbury, A. J.; Glenny, A. M.; Thakker, N. S. \& Sloan, P. Opinions and attitudes of the UK's GDPs and specialists in oral surgery, oral medicine and surgical dentistry on oral cancer screening. Oral Diseases, 12(2):194-9, 2006.

Morales-Navarro, D.; Rodríguez-Lay, L. \& García-Jordán, M. Importancia del Programa de Detección Temprana del cáncer bucal en Cuba. Revista Habanera de Ciencias Médicas, 8(4), 2009.

Morse, D. E.; Vega, C. M. V.; Psoter, W. J.; Vélez, H.; Buxó, C. J.; Baek, L. S. \& Ayendez, M. S. Perspectives of San Juan health care practitioners on the detection deficit in oral pre malignant and early cancers in Puerto Rico: a qualitative research study. BMC Public Health, 11(1):391, 2011.

M, S.; Shetty, P.; Decruz, A. M. \& Pai, P. The Self-Reported Knowledge, Attitude and the Practices Regarding the Early Detection of Oral Cancer and Precancerous Lesions among the Practising Dentists of Dakshina Kannada - APilot Study. Journal of Clinical and Diagnostic Research?: JCDR, 7(7):1491-4, 2013.

Noonan, B. Understanding the reasons why patients delay seeking treatment or oral cancer symptoms from a primary health care professional: An integrative literature review. European J. Oncology Nursing, 18(1):118-124, 2014.

Patel, K. J.; De Silva, H.L.; Tong, D. C. \& Love, R. M. Concordance Between Clinical and Histopathologic
Diagnoses of Oral Mucosal Lesions. J. Oral and Maxillofacial Surg., 69(1):125-33, 2011.

Patton, L. L.; Ashe, T. E.; Elter, J. R.; Southerland, J. H. \& Strauss, R. P. Adequacy of training in oral cancer prevention and screening as self-assessed by physicians, nurse practitioners, and dental health professionals. Oral Surgery, Oral Medicine, Oral Pathology, Oral Radiology and Endodontics, 102(6):758-64, 2006.

Reichart, P. A. \& Philipsen, H. P. Oral erythroplakia--a review. Oral Oncology, 41(6):551-61, 2005.

Rhodus, N.L; Kerr A.R. \& Patel, K. Oral Cancer?: Leukoplakia, Premalignancy, and Squamous Cell Carcinoma. Dental Clinics of North America, 58(2):315-40, 2014.

Rocha-Buelvas, A.; Hidalgo-Patiño, C.; Collela, G. \& Angelillo, I. Oral cancer and dentists: knowledge, attitudes and practices in a South Colombian context. Acta Odontológica Latinoamericana: AOL, 25(2):155-62, 2012.

Yellowitz, J. A.; Horowitz, A. M.; Drury, T. F. \& Goodman, H. S. Survey of U.S. dentists' knowledge and opinions about oral pharyngeal cancer. J. Am Dental Assoc. (1939), 131(5):653-61, 2000.

Yellowitz, J.; Horowitz, A. M.; Goodman, H. S.; Canto, M. T. \& Farooq, N. S. Knowledge, opinions and practices of general dentists regarding oral cancer: a pilot survey. $J$. Am. Dental Assoc., 129(5):579-83, 1998.

Dirección para correspondencia

Anika Stillfried

Carrera de Odontología

Facultad de Medicina

Clínica Alemana - Universidad del Desarrollo

Santiago

CHILE

Email:anika.stillfried88@gmail.com

Recibido : 26-08-2016

Aceptado: 06-12-2016 